\title{
Detection of Weak Circumstellar Gas around the DAZ White Dwarf WD 1124-293: Evidence for the Accretion of Multiple Asteroids
}

\author{
J. H. Debes ${ }^{1}$, M. Kilic ${ }^{2}$, F. Faedi ${ }^{3}$, E. L. Shkolnik ${ }^{4}$, M. Lopez-Morales ${ }^{5,6}$, A. J. Weinberger ${ }^{7}$, \\ C. Slesnick ${ }^{7,8}$, R. G. West ${ }^{9}$
}

\begin{abstract}
Single white dwarfs with photospheric metal absorption and no dusty disks are believed to be actively accreting metals from a circumstellar disk of gas caused by the disruption and sputtering of asteroids perturbed by post-main sequence planetary systems. We report, for the first time, the detection of circumstellar Ca II gas in absorption around the metal-polluted white dwarf WD 1124-293 at $>7 R_{\mathrm{WD}}$ and $<32000 \mathrm{AU}$, with a probable distance of $\sim 54 \mathrm{R}_{\mathrm{WD}}$. This detection is based on several epochs of high-resolution optical spectroscopy around

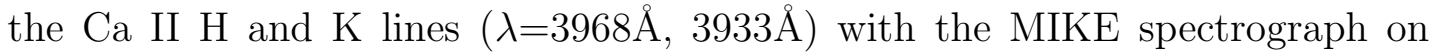
the Magellan/Clay Telescope. We confirm the circumstellar nature of the gas by observing stars with small angular separations to WD 1124-293 and larger distances from Earth-none of the reference stars show absorption consistent with
\end{abstract}

\footnotetext{
${ }^{1}$ Space Telescope Science Institute, 3700 San Martin Dr., Baltimore, MD 21218

${ }^{2}$ Homer L. Dodge Department of Physics and Astronomy, The University of Oklahoma, 440 W. Brooks St., Norman, OK 73019

${ }^{3}$ Astrophysics Research Centre, School of Mathematics and Physics, Queen's University Belfast, University Road, Belfast, BT7 1NN, UK

${ }^{4}$ Lowell Observatory, Flagstaff, AZ 86001

${ }^{5}$ Institut de Ciències de l'Espai (CSIC-IEEC), Campus UAB, Facultat de Ciències, Torre C5, parell, 2a pl, E-08193 Bellaterra, Barcelona, Spain

${ }^{6}$ Visiting Investigator, Department of Terrestrial Magnetism, Carnegie Institution of Washington, 5241 Broad Branch Road. N.W., Washington DC 20015, USA

${ }^{7}$ Department of Terrestrial Magnetism, Carnegie Institution of Washington, 5249 Broad Branch RD, N.W., Washington, DC 20015

${ }^{8}$ Charles Stark Draper Laboratory, Inc., 555 Technology Square, Cambridge MA 02139

${ }^{9}$ Department of Physics and Astronomy, University of Leicester, University Road, Leicester, LE1 7RH, U.K.
} 
the presence of appreciable local interstellar medium at the distance of the white dwarf. By combining our observations over four years with previous spectra of WD 1124-293, we have measured the equivalent width of the two photospheric Ca lines over a period of 11 years. We see $<5-7 \%$ epoch-to-epoch variation in equivalent widths over this time period, and no evidence for long term trends in the strength of the Ca II lines. Since it is likely that the circumstellar gas arises from a disk edge-on to our line of sight, we also place limits to short period transiting substellar and planetary companions with $\mathrm{R}>\mathrm{R}_{\oplus}$ using the WASP survey. The presence of gas in orbit around WD 1124-293 implies that most metal rich WDs could harbor relic planetary systems. Since roughly $25-30 \%$ of WDs show metal line absorption, the dynamical process for delivering small bodies close to a WD must be robust.

Subject headings: circumstellar matter-planetary systems-white dwarfs

\section{Introduction}

In the absence of externally accreted material, radiative levitation, or convective dredgeup, white dwarfs (WDs) should have pure hydrogen or pure helium photospheres. All other elements heavier than helium should settle out of view on timescales much shorter than the cooling age of WDs. Hydrogen WDs (type DA) have photospheric settling times that can range from days to thousands of years, while helium WDs (type DB) have settling times as long as 1 Myr. Due to their high gravity and thin photospheres, WDs are some of the most sensitive probes to low levels of external accretion. A typical $0.6 \mathrm{M}_{\odot}$ hydrogen WD with an effective temperature of $10000 \mathrm{~K}$ can show observable Ca II lines from the accretion of $\sim 10^{6} \mathrm{~g} / \mathrm{s}$ of solar abundance material, corresponding to the accretion of a small asteroid's worth of material per year (Paquette et al. 1986; Koester et al. 2005; Koester 2009). WDs are sensitive probes of the accretion from the surrounding interstellar medium (ISM) (Dupuis et al. 1992, 1993a.b), the winds from companions (Sion \& Starrfield 1984; Zuckerman et al. 2003; Debes 2006), and the accretion of asteroidal material from orbiting dust disks (e.g. Zuckerman et al. 2007; Klein et al. 2010; Vennes et al. 2010; Dufour et al. 2010; Debes et al. 2011a,b). All three of these phenomena can explain the presence of metal lines in WDs, but a significant fraction of metal polluted WDs (type DAZ for hydrogen atmosphere WDs and type DBZ or DZ for helium atmosphere WDs) are apparently single, do not possess any dust disks, and do not have sufficient surrounding ISM to explain their accretion (Aannestad et al. 1993; Kilic \& Redfield 2007).

ISM accretion was the first logical explanation for metal polluted WDs (Wesemael 1979; 
Wesemael \& Truran 1982; Dupuis et al. 1992, 1993a,b). However, studies of polluted WDs show no strong correlation between velocity and metal abundance as would be expected for Bondi-Hoyle accretion of the ISM (Aannestad et al. 1993; Zuckerman et al. 2003, 2010; Kilic \& Redfield 2007). Nor is there any correlation between metal abundance and constraints on the local ISM (Kilic \& Redfield 2007). Most observed DAZs and DZs reside well within the low density Local Bubble, and are not physically located near known dense molecular clouds. The lack of appreciable hydrogen in DZs also cannot easily be explained by the accretion of solar abundance material.

The presence of polluted DAZs that are members of WD+red dwarf binaries raises the possibility that previously unseen companions could be the cause of many polluted WDs through the accretion of a companion wind (Zuckerman et al. 2003). While it is physically plausible that a close ( $a<1 \mathrm{AU}$ ) companion can pollute a WD with its wind (Debes 2006), most WDs that have been observed with Spitzer are constrained to have no close stellar or brown dwarf companions (Mullally et al. 2008; Debes et al. 2007; Farihi et al. 2009). The most massive objects that could orbit these WDs would have masses of 5-15 $\mathrm{M}_{\text {Jup }}$.

Dusty WDs all show pollution due to the direct accretion of metal rich grains onto the WD surface. However, only $\sim 30 \%$ of metal polluted WDs show infrared excesses due to dusty disks (Kilic et al. 2008; Farihi et al. 2010). Some disks may be close to edge-on to our line of sight and hidden, but inclination effects alone cannot explain the relative number of dusty and non-dusty WDs. Tidally disrupted planetesimals perturbed by planets are believed to be the origin of dusty WDs (Debes \& Sigurdsson 2002; Jura 2003; Bonsor et al. 2011). Similarly, apparently single WDs with metal pollution may be caused by hitherto unseen disks that are either dusty, optically thin, and undetectable in the infrared, or entirely gaseous. Gaseous disks with some mass in gas of $M_{\text {disk }}$ that arise from the sputtering of debris from tidally disrupted planetesimals have been postulated to explain the relative number of dusty and non-dusty WDs (Jura 2008). In this scenario, only planetesimals larger than $\sim 2 \mathrm{M}_{\text {disk }}$ create an optically thick dusty disk, while smaller objects are completely ablated by the relative velocity between the debris and the existing gaseous disk, further populating the disk with gaseous material.

Circumstellar gas emission lines have been observed toward several hotter WDs that also possess dust disks (Gänsicke et al. 2006, 2007, 2008; Melis et al. 2010), demonstrating that significant gas can be generated from either the sublimation, collision, or photoionization of dust grains. Similarly, a handful of hotter WDs that have been observed in the UV show circumstellar gas absorption (Holberg et al. 1998; Bannister et al. 2003; Lallement et al. 2011; Dickinson et al. 2012). These hotter WDs may be evaporating circumstellar dust disks or local ISM, but to date none of these systems has been shown to possess a dust disk-their 
origins are less clear. As WDs become hotter, radiative levitation of metals becomes a more important process and can explain the presence of metals in a WD atmosphere for effective temperatures larger than $25000 \mathrm{~K}$ (Chayer \& Dupuis 2010).

In this work we present the detection at 8- $\sigma$ of weak circumstellar Ca II absorption in orbit around the cooler DAZ WD 1124-293. Based on observations of other sight lines close to WD 1124-293 and the relative velocity of the circumstellar gas, we confine the spatial extent of the absorption to $7 R_{\mathrm{WD}}<r<32000$ AU from WD 1124-293. We argue that it must arise from a circumstellar gaseous disk in orbit around the WD at a probable radius of $\sim 54 R_{\mathrm{WD}}$, well within the WD's tidal disruption radius. In $\$ 2$ we describe the MIKE spectroscopic observations of WD 1124-293 and present the results of those observations in §3. We also search WASP data for planetary transits around WD 1124-293 in \$4. We search for variability in the accretion of material on the WD in $\$ 5$ and present our conclusions in $\S$ 6.

\section{Observations}

We have taken optical spectra of WD 1124-293 over 16 separate days spanning 4 years with the MIKE spectrograph (Bernstein et al. 2003) on the Clay Telescope at Las Campanas Observatory between 2007 and 2011 (See Table 1) as part of a large DAZ monitoring project (Debes \& Kilic 2010). WD 1124-293 was first discovered in the Edinburgh-Cape Survey and listed in McCook \& Sion (1999). Based on spectral models of WDs, WD 1124293 has $\mathrm{M}=0.66 \mathrm{M}_{\odot}$ and $\mathrm{T}_{\text {eff }}=9400 \mathrm{~K}$ (Koester \& Wilken 2006), with a spectroscopically determined distance of 33.6 pc (Pauli et al. 2006). Photospheric metal lines of Ca II were detected with moderate resolution spectroscopy from Keck HIRES (R 30000) and VLT UVES (R 18500) (Zuckerman et al. 2003; Koester et al. 2005), which showed moderately strong Ca II H and K lines $(\lambda=3968.469,3933.664)$ with a heliocentrically corrected redshift of $29.5 \mathrm{~km} \mathrm{~s}^{-1}\left(29 \pm 1 \mathrm{~km} \mathrm{~s}^{-1}\right)$ for Keck (VLT) in December 1998 and April 1999 (April and May 2000). The calcium cannot be explained by accretion of the local ISM based on mea-

surements of the local ISM density (Kilic \& Redfield 2007). The equivalent width (EW) of the Ca II line over the four separate epochs with VLT and Keck averaged $\approx 106 \mathrm{~mA}$ with no significant variation over this period, implying a steady state accretion rate of $4 \times 10^{15} \mathrm{~g} / \mathrm{yr}$ (Koester \& Wilken 2006), assuming 1\% metal rich material relative to solar abundance. In January and June of 2010 we observed four bright F and A stars located near WD 1124-293 on the sky in order to probe the surrounding ISM at the distance of WD 1124-293.

For our MIKE spectra, we used a $0^{\prime \prime} .7 \times 5^{\prime \prime}$ slit which corresponded to a resolution of $\sim 40,000$ at the Ca II K line. Th-Ar comparison lamp spectra were taken near in time to 
each spectrum of WD 1124-293. In addition, we obtained the previously reduced spectra of this WD from the VLT and Keck (Koester et al. 2005; Zuckerman et al. 2003). The VLT spectra were kindly provided by D. Koester, while the Keck data was publicly available with the journal article it was published in.

Our MIKE data were flatfielded and bias-subtracted using the MIKE reduction pipeline written by D. Kelson, with methodology described in Kelson et al. (2000) and Kelson (2003). We initially extracted the spectra using the full pipeline, but discovered that the pipeline was subtracting the background at a level of precision insufficient for high quality equivalent width measurements. To rectify this, we performed our own extraction, background subtraction, and wavelength solution for $8-10 \AA$ windows centered on the $\mathrm{Ca} \mathrm{H}$ and $\mathrm{K}$ lines for all of our MIKE spectra. The spectra were also corrected for heliocentric velocity shifts between observations. Since the Ca II K line falls on two orders, we combined each order by fitting the continuum with a polynomial and averaging the orders for each night to increase signal-to-noise $(\mathrm{S} / \mathrm{N})$ to the values reported in Table 1. All the fitted spectra from MIKE were then averaged with a $\mathrm{S} / \mathrm{N}$ weighting to produce a final spectrum of the region around each line.

In order to check for variability in our spectra between 2007-2011 as well as with the previous observations, we calculated the EW of the two calcium lines from each night's spectrum. To calculate the EW, we chose a window around the line equivalent to \pm 3 times the standard deviation of the line as determined by a Gaussian fit, which also returned a measure of the radial velocity for the WD on each night. We chose several different polynomial fits to the continuum and added any systematic uncertainty in the continuum fit to our uncertainties in the EW calculation. This approach has the advantage of calculating a more accurate EW despite differences in resolution between different epochs, since the total integrated line flux is conserved from observation to observation. Table 2 details the observed photospheric line radial velocities and equivalent widths. From the individual measures of the K line with MIKE, we obtain a median radial velocity for WD $1124-293$ of $29.0 \pm 0.5 \mathrm{~km} \mathrm{~s}^{-1}$.

\section{Results}

The final, S/N weighted spectrum of WD 1124-293 at the Ca $\mathrm{H}$ and $\mathrm{K}$ lines is shown in Figure 1. Just to the blue of the main photospheric lines we detect weak lines indicative of another Ca II component, with line depths of 3.4- and 8.5- $\sigma$ below the continuum for the $\mathrm{H}$ and $\mathrm{K}$ line respectively. As seen in Table 2 , the lines at both wavelengths are separated by $\approx 30 \mathrm{~km} \mathrm{~s}^{-1}$ to the blue of the photospheric Ca II. Weak lines in the spectrum could be due to additional photospheric species, circumstellar Ca II gas, intervening ISM Ca II gas, or an 
artifact from the spectrograph or reduction process. In this section we argue that the lines arise from circumstellar Ca II gas in orbit around the WD.

\subsection{Ruling out artifacts and other atomic species}

There are three separate conditions that rule out the possibility that the lines could arise from the data reduction process or the MIKE spectrograph. First, we have verified that the line near the photospheric Ca II K line was detected significantly on a $\mathrm{S} / \mathrm{N}$ combined spectrum of each of the two echelle orders that the line fell on. The position of the line on the two orders is separated by $\approx 1100$ pixels, ruling out possible artifacts from the instrument. For both orders, the line is detected at the proper position and at 4- and 6- $\sigma$ below the continuum. Secondly, the line is detected significantly in the final combined spectra when using the full Kelson MIKE pipeline, which used different methodology to perform order extraction and background subtraction. Finally, when we combine the data into subsets, we recover the line as well. Figure 2 shows three separate epochs: March 2008, May 2008, and a combination of our June 2010 and June 2011 data. In particular, the heliocentric velocity correction in March 2008 and our June 2010+2011 data differed by $23 \mathrm{~km} \mathrm{~s}^{-1}$, ruling out any terrestrial origin for the line, since the epoch-to-epoch centers match to within $0.01 \AA$.

These lines could be weak photospheric features. We searched atomic line lists, as well as metal lines other than calcium reported for other polluted WDs (e.g. Klein et al. 2010), and found no atomic lines that could plausibly be matched to the observed spectrum of WD 1124-293. Furthermore, the line depths and relative equivalent widths are consistent, within the uncertainties, to the expected line strength ratio between the Ca II $\mathrm{H}$ and $\mathrm{K}$ lines. Based on these arguments, the weak features must arise from intervening ISM or circumstellar Ca II gas.

\subsection{Ruling out Local Interstellar Absorption}

While ISM absorption could explain the presence of the weak features we observe, we rule out an ISM contribution. The Sun resides in a low-density ISM structure known as the Local Bubble. WD 1124-293 is at a spectroscopically determined distance of $33.6 \mathrm{pc}$, well inside the Local Bubble (Pauli et al. 2006). The local interstellar medium is relatively devoid of cold neutral gas, until the accumulation of dense material at $\sim 80 \mathrm{pc}$ (Welsh et al. 2010). However, warm, partially ionized clouds have been detected through weak Ca II ISM absorption features in the spectra of stars within 50 pc of the Sun (see Redfield \& Linsky 
2008; Welsh et al. 2010, for a review of the properties of the local interstellar medium).

If the weak Ca II absorption feature in WD 1124-293 is due to ISM accretion, other stars in close proximity on the sky should also show a similar absorption feature. In order to test this possibility, we observed several bright stars at angular separations $<1.6^{\circ}$ from WD 1124-293 to search for absorption features at the same velocity. This technique, as well as searching for line variability, has been used in the past to confirm circumstellar absorption around HD 32297, a young star with an edge-on debris disk (Redfield 2007). We observed four stars with constraints on their distance from Hipparcos and with the same setup as WD 1124-293: HIP $55864\left(\mathrm{~F} 5 \mathrm{~V}, \mathrm{r}=0.27^{\circ}\right)$ HIP 55901 (A0V, $\left.\mathrm{r}=0.71^{\circ}\right)$, HIP $55731\left(\mathrm{~A} 9 \mathrm{~V}, \mathrm{r}=1.10^{\circ}\right)$, and HIP 55968 (A3V, r=1.58 $)$ (See Table 1). Another star, HIP 56280A (F8V, r=1.19 ${ }^{\circ}$ ), had a spectrum that was kindly provided to us by S. Desidera. Figure 3 shows how each ISM standard is arranged on the sky relative to WD 1124-293.

The distances for each star are given in the latest reduction of Hipparcos data (van Leeuwen 2007). HIP 56280A and HIP 55864 have secure distances (parallax measurements with errors of $<20 \%$ ), while HIP 55731 and HIP 55901 have marginally detected parallaxes $(\sim 3-\sigma)$. HIP 55968's parallax is consistent with zero. For the marginal detections, we take as the stellar distance the 3- $\sigma$ upper limit to the parallax, which is 6.62 mas (151 pc) and 5.67 mas (176 pc) for HIP 55731 and HIP 55901 respectively. HIP 55968's optical and NIR magnitudes and spectral type are consistent with this star lying between HIP 55731 and HIP 55901, so we take the lower limit to its distance to be $151 \mathrm{pc}$.

Each star had broad Ca II K photospheric absorption which we removed by applying a polynomial fit to the photospheric line, an example of which is shown in Figure 4 . For HIP 56280A, its narrow line core was fitted with two polynomials joined at the line core center of $3933.704 \AA$. The resulting continuum fitted spectra were inspected for narrow absorption components. HIP 56280A (to lower significance) and HIP 55864 show no appreciable absorption features comparable in strength to the WD 1124-293 line, while HIP 55731 shows a weak (presumably ISM) component far from the radial velocity or our observed feature. HIP 55901 and HIP 55968, both constrained by Hipparcos to be more distant than HIP 55864, each showed absorption from at least three ISM components. We attempted to simultaneously fit these three components for the two stars and present our best fits in Figure 5, which shows WD 1124-293's Ca II K line region compared with our ISM standards. Table 3 shows the resulting velocity components for these fits, consistent with at least three distinct complexes of ISM gas that slowly vary on size scales of $\sim 1-2 \mathrm{pc}$, one of which is at a radial velocity within a few $\mathrm{km} \mathrm{s}^{-1}$ of the circumstellar feature. Despite the similar radial velocity of the two features to what is observed in WD 1124-293, the non-detection of any similar lines for HIP 56280, HIP 55864, and HIP 55731 constrains the location of these ISM 
components to be at a distance $>151$ pc. The non-detection of the faint candidate circumstellar component for HIP 55864 constrains the extent of the Ca II gas near WD 1124-293 to $<0.27^{\circ}$ at $\mathrm{d}=33.6 \mathrm{pc}$, or roughly $32000 \mathrm{AU}(0.16 \mathrm{pc})$.

Welsh et al. (2010) find a slowly increasing Ca II equivalent width with increasing distance, but depending on whether local gas clouds are encountered or not, they find both low and high values of $\mathrm{Ca}$ II volume density within $30 \mathrm{pc}$. Out of the 1857 stars analyzed by Welsh et al. (2010), 50 are within 40 pc of the Sun. Only one of these 50 stars, HD 159561 $\left(l=36^{\circ}, b=+23^{\circ}\right)$, shows an ISM absorption feature with a Ca II equivalent width stronger than what is observed for the weak feature in WD1124-293 $\left(l=282^{\circ}, b=+30^{\circ}\right)$. The ISM complexes observed for our more distant stars are consistent with a relatively dense cloud of material that lies $>90$ pc along the Galactic sightline to these stars (Welsh et al. 2010), and thus cannot account for the weak Ca II feature we measure for WD 1124-293. Hence, it is unlikely that this feature is due to an intervening warm ISM cloudlet. The only scenario that the data allows is if an extremely narrow warm ISM column near WD 1124-293 (width $\sim 0.06 \mathrm{pc}$ and height $\sim 1.3 \mathrm{pc}$ ) with a velocity gradient intersected the sightlines to WD 1124-293, HIP 55901, and HIP 55968, but did not intersect the sightline of HIP 55864.

Such a pathological structure is not supported by studies of the nearby ISM. Redfield \& Linsky (2001) studied the small scale (0.05-1.2 pc) structure of the local interstellar cloud and find that the $\mathrm{Mg}$ II column densities do not vary by more than a factor of two for $\leq 0.6 \mathrm{pc}$ scales. The implied Ca II column densities by the much stronger ISM absorption observed towards HIP 55901 and HIP 55968 are an order of magnitude larger than for WD 1124-293, and would have been easily detected in the ISM standards that showed no component at the velocity of our candidate circumstellar feature. Hence, the observations of our ISM standards limits the amount of interstellar Ca II between WD 1124-293 and us at the velocity of the Ca II $\mathrm{K}$ feature to $\mathrm{EW}<6 \mathrm{~m} \AA$.

The source of metals in high surface gravity WDs has been a puzzle for decades (see Dupuis et al. 1993a). The search for a correlation between the local ISM clouds and the locations of metal-rich WDs was inconclusive (Zuckerman et al. 2003). Kilic \& Redfield (2007) used the observed ISM column densities toward stars in close proximity to the known DAZ WDs to demonstrate that there is no correlation between the accretion density required to supply metals observed in DAZs with the densities observed in their interstellar environment, including WD 1124-293. Therefore, the DAZ WD population as a whole argues against ISM accretion being the dominant contributor to the metals in WDs.

In conclusion, it is more likely that the weak Ca II feature is due to circumstellar gas in orbit around WD1124-293 because (1) no similar absorption features are detected in neighboring sightlines as close as $0.16 \mathrm{pc}$ and the local interstellar medium is relatively 
homogeneous at such scales (Redfield \& Linsky 2001), (2) the strength of the absorption feature is uncommon for stars within 40-50 pc of the Sun, (3) the absorption feature matches the stellar radial velocity, and (4) there is no correlation between the metal abundances of known DAZ stars and the ISM densities (Kilic \& Redfield 2007).

\subsection{Limits to the inner extent of circumstellar gas}

If the line arises from gas in orbit around the WD, the relative velocity between the photospheric line and the circumstellar feature can be used to determine how deep the gas lies in the gravitational well of the WD. The photospheric Ca II line is offset from the true systemic velocity of the WD due to the gravitational redshift of WD 1124-293. Using the latest values of WD 1124-293's $\log \mathrm{g}=8.096$ and its $\mathrm{T}_{\mathrm{eff}}=9420 \pm 150 \mathrm{~K}$ (D. Koester, private communication), we have calculated masses from cooling models of WD\&1 (Bergeron et al. 1995; Holberg \& Bergeron 2006; Tremblav et al. 2011; Bergeron et al. 2011) which use the massradius relation of Fontaine et al. (2001) to determine the gravitational redshift of WD 1124293. The uncertainty in the gravity of the WD dominates the uncertainty in the expected gravitational redshift, but is also not well known. To estimate this possible uncertainty we compare our value to that given in Koester et al. (2001) and Koester (2009), which vary between 8.04 to 8.099. We therefore estimate the systematic uncertainty in the WD gravity to be $\approx 0.06$. Using this value and uncertainty and calculating a gravitational redshift $\left(v_{\text {grav }}\right)$, we obtain $v_{\text {grav }}=34.9 \pm 3.7 \mathrm{~km} \mathrm{~s}^{-1}$, implying a systemic velocity $\gamma=-5.9 \pm 3.7 \mathrm{~km} \mathrm{~s}^{-1}$

Previous calculations of WD 1124-293's $\gamma$ (based on its inferred mass and radius from synthetic spectral modeling) find $-2.7 \pm 4.3 \mathrm{~km} \mathrm{~s}^{-1}$, consistent with our calculated $\gamma$ (Pauli et al. 2003, 2006). The primary difference between our result and previous calculations of $\gamma$ come from a higher WD gravity (The earlier values of $\log g$ being 0.05 dex lower), highlighting the dependence of this calculation on a detailed knowledge of a WD's properties. The measured relative velocity between the photospheric line and the circumstellar line $\left(v_{\text {gas }}\right)$ from our $\mathrm{S} / \mathrm{N}$ weighted spectrum is $-29.9 \pm 0.8 \mathrm{~km} \mathrm{~s}^{-1}$. The orbital radius of the gas is then

$R_{g}=v_{\text {grav }} /\left(v_{\text {gas }}+v_{\text {grav }}\right) \mathrm{R}_{\mathrm{WD}}$, implying a minimum radius to the gas of $7_{-3}^{+11} R_{\mathrm{WD}}$. Given the uncertainties, the faint line could correspond to the systemic velocity and reside further from the WD, thus directly probing the gravitational redshift of the WD. This minimum radius then should be treated as a lower limit to the gas radius. For optically thin silicate dust, the sublimation radius is given by (Jura 2008; Kimura et al. 2002; Hoard 2011):

\footnotetext{
${ }^{1}$ These models are available at http://www.astro.umontreal.ca/ bergeron/CoolingModels
} 


$$
R_{\mathrm{sub}}=3.7 \times 10^{10}\left(\frac{L_{\mathrm{WD}}}{0.001 L_{\odot}}\right)^{1 / 2}\left(\frac{1000 K}{T_{\mathrm{sub}}}\right)^{2} \mathrm{~cm} .
$$

From the cooling models used to determine WD 1124-293's gravitational redshift, we also determine a luminosity of almost exactly $0.001 L_{\odot}$, leading to a sublimation radius of $\approx 44 R_{\mathrm{WD}}$, which is close to the inferred radius of the gas from kinematic arguments in $\$ 3.4$.

\subsection{Properties and Origin of the Circumstellar Gas}

Further characterization of the Ca II lines permits additional constraints on the origin and properties of the circumstellar gas around WD 1124-293. In particular, the different constraints point to a localized disk of gas a few tens of WD radii from WD 1124-293 and well within its tidal disruption radius.

In order to determine the column density of the Ca II gas, we minimized a $\chi^{2}$ metric for an optically thin gas column by varying the column density $(\log N)$ and FWHM of the line, using the proper oscillator strengths and assuming a Gaussian line profile. From the minimization we find a best fitting line model with (98\% confidence) $\log N=11_{-0.2}^{+0.1} \mathrm{~cm}^{-2}$ and $\mathrm{FWHM}=0.2 \pm 0.1 \AA$ with a $\chi_{\nu}^{2}=1.08$. The best fit line, compared to the observed circumstellar line is shown in Figure 6. The model overpredicts the expected line strength of the Ca II H line, but given the low $\mathrm{S} / \mathrm{N}$ of the line it is hard to determine if this difference is significant. Since this is the ionized state of calcium, it is impossible to tell how much total gas resides in the system without a) other gas species or b) a measurement of Ca I to determine the temperature and electron density of the gas. Further characterization of the gas will be the focus of future work, but is beyond the scope of this paper.

We can also constrain the location of the gas by the width of the weak Ca II line-an upper limit to the orbital velocity of the gas can be determined from the line FWHM, and thus a characteristic radius for the disk. We assume that the gas is in a circular orbit and that the line FWHM corresponds to the range of orbital radial velocities within the circumstellar gaseous disk that intersects the angular disk of the WD on the sky:

$$
r_{\mathrm{disk}} \approx\left(G M_{\mathrm{WD}}\right)^{1 / 3}\left[\frac{2 R_{\mathrm{WD}}}{F W H M\left(\mathrm{~cm} \mathrm{~s}^{-1}\right)}\right]^{2 / 3}
$$

From our gravitational redshift calculations, $R_{\mathrm{WD}} \approx 8.4 \times 10^{8} \mathrm{~cm}$, and the FWHM of the line is $16 \mathrm{~km} \mathrm{~s}^{-1}$, giving a radius for the disk of $4.5 \times 10^{10} \mathrm{~cm}$ or $\approx 54 R_{\mathrm{WD}}$. This is consistent 
with the gas being close to the WD and within the tidal disruption radius of WD 1124-293, as well as being from the sublimation of optically thin dust.

The constraints on the location of the gas and the presence of Ca II combined with the lack of any appreciable ISM all point to a similar origin for WD 1124-293 as that posited for WDs with near-IR excesses due to dusty disks. These observations fit in with the scenario where dusty excesses are caused by massive asteroids or comets that are dynamically perturbed by a planetary system (Debes \& Sigurdsson 2002; Bonsor et al. 2011; Bonsor \& Wyatt 2012; Debes et al. 2012) and then tidally disrupted (Jura 2003). However, a size distribution of planetesimals will be disrupted over time, and for smaller bodies, sputtering renders most tidally disrupted dust into a gaseous phase quite quickly, forming

circumstellar gas disks instead of circumstellar dust disks (Jura 2008; Farihi et al. 2012). Circumstellar gas absorption roughly at the same radius as that observed for dusty disks around WDs is also suggestive of a tidally disrupted asteroidal origin for our observations of WD 1124-293.

\section{Limits to Transiting Companions to WD 1124-293}

Given the possible edge-on inclination of this system due to the presence of circumstellar gas, the probability of any detectable planetary companion transiting WD 1124-293 might be higher than a randomly inclined system. To that end we have gathered all data of WD 1124293 from the Wide Angle Search for Planets (WASP) transit database. From previous observations, WD 1124-293 does not possess close-in brown dwarf or stellar companions. In their comprehensive Spitzer search for IR excesses due to companions around a sample of WDs, Farihi et al. (2008) placed a definitive upper limit of $12 \mathrm{M}_{\text {Jup }}$ to the presence of any unresolved companions in orbit around WD 1124-293.

The WASP North and South telescopes are located in La Palma (ING - Canary Islands) and Sutherland (SAAO - South Africa), respectively. Each telescope consists of 8 Canon $200 \mathrm{~mm} f / 1.8$ focal lenses coupled to e2v $2048 \times 2048$ pixel CCDs, yielding a field-of-view of $7.8 \times 7.8$ square degrees with a pixel scale of $13.7^{\prime \prime}$ (Pollacco et al. 2006). The WASP observing strategy is such that each field is observed with a typical cadence of the order of 8-10 minutes and typical exposures of 1 minute (30 sec exposure plus over heads). WASP provides good quality photometry with a precision exceeding $1 \%$ per observation in the approximate magnitude range $9 \leq \mathrm{V} \leq 12$.

WD 1124-293 was observed by the WASP-South telescope in the 2007 and 2008 seasons covering the interval 2007 January 04 to 2007 June 05, and 2008 January 05 to 2008 May 
28, respectively. The 10618 pipeline-processed photometric measurements were de-trended using the Tamuz algorithm (Tamuz et al. 2005) to account for linearly-correlated systematic noise in the data. We show the WASP light-curve in Figure 7 (bottom-panel).

We used the modified implementation of the Box-Least Square (BLS) algorithm described in Faedi et al. (2011) to search for transits and eclipses of sub-stellar and planetary companions in close orbits around the white dwarf WD1124-293. We searched a parameter space defined by orbital periods ranging from 2 hours to 15 days, and companion sizes ranging from Moon-size to Jupiter-size. No transiting planet has been identified. Figure 6 (top-panel), shows the BLS power-spectrum for the star WD1124-293. The dashed-line indicates the detection threshold as defined in Faedi et al. (2011) for $10 \%$ noise levels. We have phase-folded the WASP light-curve to investigate the possible presence of an astrophysical signal at the periods detected above the threshold. However, none showed real variation. Finally, we have used the modified Lomb-Scargle periodogram (Scargle 1982) to search for generic variability. We find that a real power is assigned to a peak at $\mathrm{P}=27.42$ days which is most probably related to the Moon cycle. We used these null results together with the results of our simulations to estimate an upper-limit to the frequency of close companions to WD 1124-293.

To assess the chances of detecting eclipses and transits of substellar and planetary companions to WD1124-293, we performed an extensive set of Monte Carlo simulations. The approach we adopted was to create realistic synthetic light-curves containing eclipse and transit signatures of the expected depth $\left(\delta_{\mathrm{tr}}\right)$ and duration for a range of companion sizes and orbital periods, then to attempt to detect these signatures using the modified BLS mentioned above. By noting the rate at which the BLS search recovered the transit $\left(f_{\text {det }}\right)$ at the correct period (or an integer multiple or fraction) we were able to estimate the detection limits of such systems in an automated manner.

Details of the synthetic transit signal generated, the corresponding transit probability, depth and duration are described in detail in Faedi et al. (2011). Here we present the results of simulations covering the orbital period-planet radius parameter space defined by seven trial periods spaced approximately logarithmically $(P=0.08,0.22,0.87,1.56,3.57,8.30$ and 14.72 days), and five planet radii $R_{\mathrm{p}}=10.0,4.0,1.0,0.55$, and $0.27 \mathrm{R}_{\oplus}$.

Figure 8 shows the detection rate of the simulated transit signals injected in the WASP light curve of WD1124-293. We regard as a match any trial in which the most significant detected period is within $1 \%$ of being an integer fraction or multiple from $1 / 5 \times$ to $5 \times$ the injected transit signal. From our result on the simulations conducted using the WASP light curve of WD1124-293 (Figure 8, see also results on simulations conducted on WASP data detailed in Faedi et al. 2011), we would have been able to detect the transit signal due to 
a companion of radius $1 \mathrm{R}_{\oplus}$ and period between 2 and $16 \mathrm{~h}$ with a confidence of $75 \%$. For objects of radius similar to that of the Earth but slightly bigger (e.g. up to $3 \mathrm{R}_{\oplus}$, or so-called SuperEarths) we would have been able to detect the eclipse signal for periods between $2 \mathrm{~h}$ and $12 \mathrm{~h}$ with a confidence of $95 \%$ and to about $1 \mathrm{~d}$ with $75 \%$ confidence. Eclipsing gas giant planets and substellar objects would have been detectable at orbital periods between $2 \mathrm{~h}$ and $1.7 \mathrm{~d}$ with $95 \%$ confidence, and with orbital periods of $2 \mathrm{~d}$ with $75 \%$ confidence. On the other end, no stringent constraint can be placed on objects with radius $<1 \mathrm{R}_{\oplus}$ even at short periods. The same is true even for large bodies such as Jupiter with orbital periods longer 2 days. From Figures 1 and 2 of Agol (2011) and using the white dwarf temperature of $9420 \mathrm{~K}$ and mass of $0.66 \mathrm{M}_{\odot}$ we find that for WD1124-293 the white dwarf habitable zone (WDHZ) extends for a region comprised between 0.02 and $0.045 \mathrm{AU}$ for which, in our simulations, we can only put weak constraints for Neptune/Uranus-size $\left(\sim 3-4 \mathrm{R}_{\oplus}\right)$ and larger objects. However, eventually WD 1124-293 will cool further and when it cools to below $7000-9000 \mathrm{~K}$, planets with orbital separations of $5 \times 10^{-3}-0.02 \mathrm{AU}$ (P 0.16-1.3 days) will be in a "continuous habitable zone" which lasts for $>3$ Gyr (Agol 2011). Our observations thus rule out a significant fraction of $>$ Earth-sized planets in this system that might reside in the continuous habitable zone of WD 1123-293.

We investigated the various factors which affect the efficiency of detection of these transit signals. When generating each synthetic light-curve we can easily assess a priori whether it will fail the tests requiring a minimum number of individual transits $(>5)$ and in-transit data points $(>5)$. We find that our requirements alone render transiting companions essentially undetectable at our longest trial periods (8.30 and 14.72 days); the transits are too short in duration and too infrequent to be adequately sampled. For companions around $1 \mathrm{R}_{\oplus}$ and larger however there is a good chance of detection out to periods of $\approx 2$ days.

\section{Limits to a variable Accretion Rate to WD 1124-293}

Given the stochastic origins to dust around WDs, it might be possible to observe variability in WD accretion rates. Ca variability was claimed to be present in the dusty DAZ G 29-38 (von Hippel \& Thompson 2007), but disputed with other observations (Debes \& López-Morales 2008). Discrepant abundances of Si between optical and UV observations of some dusty WDs may suggest possible variable accretion (Gaensicke et al. 2012). The multiple spectra of WD 1124-293 allow a careful look at the variability in the accretion rate of material onto the surface of the WD through EW measurements of its photospheric Ca II lines. WD 1124293's metal settling timescale for calcium is $194 \mathrm{yr}$, much longer than the total time high quality spectra of the Ca line have been available, but short enough that small changes due 
to settling may be observed. Similarly, if there were any short duration accretion events that briefly increased the accretion rate, we may have observed a sudden increase in the Ca EW.

Figure 8 shows the EW as a function of Julian date (JD). Two measurements of the Ca II K line, from May 2009 and July 2010, differ significantly from the other measurements. Similarly, the Ca II H line measurements from 1998 and March 2007 also deviate significantly. The cause of these discrepancies most likely due to lower $\mathrm{S} / \mathrm{N}$ and poor seeing, but given the good agreement between the other EW measurements and the lack of similar variability at the same time from the complementary Ca II line, we neglect these measurements in our analysis. To determine whether WD 1124-293's Ca II line strength was varying, we calculated a $\chi^{2}$ value for a constant value EW, determined from the median value of the observed EWs. With this measure, the Ca EW has a $\chi^{2}$ value of 23 (14) for the Ca II K (H) line, which corresponds to a probability that the EW is constant to $0.03(0.3)$. Based on our full survey of other DAZs for variability (Debes \& Kilic 2010), we require the probability of constant EW to be less than $10^{-3}$ to be considered significantly variable. The standard deviation of our MIKE measurements, neglecting the anomalous measurements, is $4.5 \%$ for the $\mathrm{K}$ line and $7 \%$ for the $\mathrm{H}$ line.

The roughly constant flow of material over $\sim 11$ years for this system places a firm lower limit on the amount of metal-rich material recently deposited, $4.6 \times 10^{16} \mathrm{~g}$, once again assuming metal rich material at $1 \%$ the solar abundance values, and a total amount of material over one settling time of $9 \times 10^{18} \mathrm{~g}$ assuming a settling time of $194 \mathrm{yr}$ (Koester et al. 2005). These masses correspond to asteroids with sizes of 1.5 and $9 \mathrm{~km}$ respectively, assuming a bulk density of $3 \mathrm{~g} / \mathrm{cm}^{3}$. These radii are comparable to the sizes of asteroids that should survive post-main sequence evolution at an orbital radius of a few AU (Jura 2008).

\section{Conclusions}

We have obtained moderate resolution optical spectroscopy of the DAZ WD 1124-293 over several epochs and found a weak circumstellar absorption feature of Ca II consistent with a tenuous gaseous disk supplied by small rocky bodies. Steady state accretion of material onto the WD surface appears to be occurring despite the relatively longer inferred settling timescale for metals, consistent with a recent disruption of an asteroidal body that was relatively small.

WD 1124-293 has also been searched for transiting companions given that any planetary system is nearly edge-on to our line of sight, though none are found for short periods. Our results do not rule out the possibility for planetary companions at wider separations. 
Emission from gaseous disks have been observed around metal enriched WDs with disks that have been actively accreting dust (Gänsicke et al. 2006, 2007, 2008). The origin of that gas could arise from the viscous heating of material (Werner et al. 2009) or from the photoionization of gas (Melis et al. 2010). However, these systems may be unusual in the fact that they may represent an early stage in the creation of a dusty disk or represent a massive example of a dusty disk in orbit around a WD. The accretion of many small planetesimals should proceed with little observable dust, and a primarily gaseous circumstellar disk (Jura 2008). These properties match WD 1124-293, representing the first example of a single metal enriched WD with a direct link to the tidal disruption of planetesimals and thus to a relic planetary system. The large fraction of single WDs that show metal pollution implies a large fraction of post-main sequence objects may house remnant planetary systems with a robust mechanism for delivering small objects in WD-grazing orbits. A large spectroscopic survey of apparently single, dust-free WDs with high $\mathrm{S} / \mathrm{N}$ may reveal more systems similar to WD 1124-293.

We wish to thank Detlev Koester for unceasing aid in discussing the fundamental parameters of WD 1124-293, and Silvano Desidera for kindly providing valuable spectra of HIP 56280. We also wish to thank Jean-Rene Roy for discussions on the kinematic broadening of spectral absorption lines, which provided a key impetus for constraining the location of WD 1124-293's gaseous disk. Finally, we want to thank the anonymous referee, whose attention to detail greatly increased the quality of this paper. This work made significant use of the SIMBAD and VIZIER databases, operated at CDS, Strasbourg, France.

\section{REFERENCES}

Aannestad, P. A., Kenyon, S. J., Hammond, G. L., \& Sion, E. M. 1993, AJ, 105, 1033

Agol, E. 2011, ApJ, 731, L31

Bannister, N. P., Barstow, M. A., Holberg, J. B., \& Bruhweiler, F. C. 2003, MNRAS, 341, 477

Bergeron, P., Wesemael, F., \& Beauchamp, A. 1995, PASP, 107, 1047

Bergeron, P., Wesemael, F., Dufour, P., Beauchamp, A., Hunter, C., Saffer, R. A., Gianninas, A., Ruiz, M. T., Limoges, M.-M., Dufour, P., Fontaine, G., \& Liebert, J. 2011, ApJ, 737,28 
Bernstein, R., Shectman, S. A., Gunnels, S. M., Mochnacki, S., \& Athey, A. E. 2003, in Society of Photo-Optical Instrumentation Engineers (SPIE) Conference Series, Vol. 4841, Society of Photo-Optical Instrumentation Engineers (SPIE) Conference Series, ed. M. Iye \& A. F. M. Moorwood, 1694-1704

Bonsor, A., Mustill, A. J., \& Wyatt, M. C. 2011, MNRAS, 414, 930

Bonsor, A. \& Wyatt, M. C. 2012, MNRAS, 420, 2990

Chayer, P. \& Dupuis, J. 2010, in American Institute of Physics Conference Series, Vol. 1273, American Institute of Physics Conference Series, ed. K. Werner \& T. Rauch, 394-399

Debes, J. H. 2006, ApJ, 652, 636

Debes, J. H., Hoard, D. W., Kilic, M., Wachter, S., Leisawitz, D. T., Cohen, M., Kirkpatrick, J. D., \& Griffith, R. L. 2011a, ApJ, 729, 4

Debes, J. H., Hoard, D. W., Wachter, S., Leisawitz, D. T., \& Cohen, M. 2011b, ApJS, 197, 38

Debes, J. H. \& Kilic, M. 2010, in American Institute of Physics Conference Series, Vol. 1273, American Institute of Physics Conference Series, ed. K. Werner \& T. Rauch, 488-491

Debes, J. H. \& López-Morales, M. 2008, ApJ, 677, L43

Debes, J. H. \& Sigurdsson, S. 2002, ApJ, 572, 556

Debes, J. H., Sigurdsson, S., \& Hansen, B. 2007, AJ, 134, 1662

Debes, J. H., Walsh, K. J., \& Stark, C. 2012, ApJ, 747, 148

Dickinson, N. J., Barstow, M. A., Welsh, B. Y., Burleigh, M., Farihi, J., Redfield, S., \& Unglaub, K. 2012, ArXiv e-prints

Dufour, P., Kilic, M., Fontaine, G., Bergeron, P., Lachapelle, F.-R., Kleinman, S. J., \& Leggett, S. K. 2010, ApJ, 719, 803

Dupuis, J., Fontaine, G., Pelletier, C., \& Wesemael, F. 1992, ApJS, 82, 505

-. 1993a, ApJS, 84, 73

Dupuis, J., Fontaine, G., \& Wesemael, F. 1993b, ApJS, 87, 345

Faedi, F., West, R. G., Burleigh, M. R., Goad, M. R., \& Hebb, L. 2011, MNRAS, 410, 899 
Farihi, J., Becklin, E. E., \& Zuckerman, B. 2008, ApJ, 681, 1470

Farihi, J., Gänsicke, B. T., Wyatt, M. A., Girven, J., Pringle, J. E., \& King, A. R. 2012, ArXiv e-prints

Farihi, J., Jura, M., Lee, J., \& Zuckerman, B. 2010, ApJ, 714, 1386

Farihi, J., Jura, M., \& Zuckerman, B. 2009, ApJ, 694, 805

Fontaine, G., Brassard, P., \& Bergeron, P. 2001, PASP, 113, 409

Gaensicke, B. T., Koester, D., Farihi, J., Girven, J., Parsons, S. G., \& Breedt, E. 2012, ArXiv e-prints

Gänsicke, B. T., Koester, D., Marsh, T. R., Rebassa-Mansergas, A., \& Southworth, J. 2008, MNRAS, 391, L103

Gänsicke, B. T., Marsh, T. R., \& Southworth, J. 2007, MNRAS, 380, L35

Gänsicke, B. T., Marsh, T. R., Southworth, J., \& Rebassa-Mansergas, A. 2006, Science, 314, 1908

Hoard, D. W. 2011, White Dwarf Atmospheres and Circumstellar Environments, ed. Hoard, D. W.

Holberg, J. B., Barstow, M. A., \& Sion, E. M. 1998, ApJS, 119, 207

Holberg, J. B. \& Bergeron, P. 2006, AJ, 132, 1221

Jura, M. 2003, ApJ, 584, L91

-. 2008, AJ, 135, 1785

Kelson, D. D. 2003, PASP, 115, 688

Kelson, D. D., Illingworth, G. D., van Dokkum, P. G., \& Franx, M. 2000, ApJ, 531, 159

Kilic, M., Farihi, J., Nitta, A., \& Leggett, S. K. 2008, AJ, 136, 111

Kilic, M. \& Redfield, S. 2007, ApJ, 660, 641

Kimura, H., Mann, I., Biesecker, D. A., \& Jessberger, E. K. 2002, Icarus, 159, 529

Klein, B., Jura, M., Koester, D., Zuckerman, B., \& Melis, C. 2010, ApJ, 709, 950

Koester, D. 2009, A\&A, 498, 517 
Koester, D., Napiwotzki, R., Christlieb, N., Drechsel, H., Hagen, H.-J., Heber, U., Homeier, D., Karl, C., Leibundgut, B., Moehler, S., Nelemans, G., Pauli, E.-M., Reimers, D., Renzini, A., \& Yungelson, L. 2001, A\&A, 378, 556

Koester, D., Rollenhagen, K., Napiwotzki, R., Voss, B., Christlieb, N., Homeier, D., \& Reimers, D. 2005, A\&A, 432, 1025

Koester, D. \& Wilken, D. 2006, A\&A, 453, 1051

Lallement, R., Welsh, B. Y., Barstow, M. A., \& Casewell, S. L. 2011, A\&A, 533, A140

McCook, G. P. \& Sion, E. M. 1999, ApJS, 121, 1

Melis, C., Jura, M., Albert, L., Klein, B., \& Zuckerman, B. 2010, ApJ, 722, 1078

Mullally, F., Winget, D. E., De Gennaro, S., Jeffery, E., Thompson, S. E., Chandler, D., \& Kepler, S. O. 2008, ApJ, 676, 573

Paquette, C., Pelletier, C., Fontaine, G., \& Michaud, G. 1986, ApJS, 61, 197

Pauli, E., Napiwotzki, R., Altmann, M., Heber, U., Odenkirchen, M., \& Kerber, F. 2003, A\&A, 400, 877

Pauli, E., Napiwotzki, R., Heber, U., Altmann, M., \& Odenkirchen, M. 2006, A\&A, 447, 173

Pollacco, D. L. et al. 2006, PASP, 118, 1407

Redfield, S. 2007, ApJ, 656, L97

Redfield, S. \& Linsky, J. L. 2001, ApJ, 551, 413

-. 2008, ApJ, 673, 283

Scargle, J. D. 1982, ApJ, 263, 835

Sion, E. M. \& Starrfield, S. G. 1984, ApJ, 286, 760

Tamuz, O., Mazeh, T., \& Zucker, S. 2005, MNRAS, 356, 1466

Tremblay, P.-E., Bergeron, P., \& Gianninas, A. 2011, ApJ, 730, 128

van Leeuwen, F. 2007, A\&A, 474, 653

Vennes, S., Kawka, A., \& Németh, P. 2010, MNRAS, 404, L40

von Hippel, T. \& Thompson, S. E. 2007, ApJ, 661, 477 
Welsh, B. Y., Lallement, R., Vergely, J.-L., \& Raimond, S. 2010, A\&A, 510, A54

Werner, K., Nagel, T., \& Rauch, T. 2009, Journal of Physics Conference Series, 172, 012054

Wesemael, F. 1979, A\&A, 72, 104

Wesemael, F. \& Truran, J. W. 1982, ApJ, 260, 807

Zuckerman, B., Koester, D., Melis, C., Hansen, B. M., \& Jura, M. 2007, ApJ, 671, 872

Zuckerman, B., Koester, D., Reid, I. N., \& Hünsch, M. 2003, ApJ, 596, 477

Zuckerman, B., Melis, C., Klein, B., Koester, D., \& Jura, M. 2010, ApJ, 722, 725 
Table 1. Spectroscopic Observations

\begin{tabular}{lcccc}
\hline \hline UT Date (JD-5400000) & Target & Observing Setup & S/N/pixel (@Ca II) & Notes \\
\hline 1998-12-12 (51159.629) & WD 1124-293 & Keck/HiRES & 37 & $\mathrm{R}=30,000$ \\
$1999-4-19(51288.328)$ & WD 1124-293 & Keck/HiRES & 26 & $\mathrm{R}=30,000$ \\
2000-4-23 (51657.663) & WD 1124-293 & VLT/UVES & 36 & $\mathrm{R}=18500$ \\
$2000-5-19(51681.588)$ & WD 1124-293 & VLT/UVES & 14 & $\mathrm{R}=18500$ \\
$2007-3-31(54189.682)$ & WD 1124-293 & Clay/MIKE & 24 & \\
$2008-3-21(54546.605)$ & WD 1124-293 & Clay/MIKE & 50 & \\
$2008-3-22(54547.656)$ & WD 1124-293 & Clay/MIKE & 50 & \\
$2008-5-13(54599.536)$ & WD 1124-293 & Clay/MIKE & 60 & \\
$2008-5-14(54600.551)$ & WD 1124-293 & Clay/MIKE & 49 & \\
$2008-5-15(54601.477)$ & WD 1124-293 & Clay/MIKE & 52 & \\
$2008-5-16(54602.502)$ & WD 1124-293 & Clay/MIKE & 56 & \\
$2009-4-16(54937.686)$ & WD 1124-293 & Clay/MIKE & 39 & \\
$2009-5-18(54969.548)$ & WD 1124-293 & Clay/MIKE & 38 & \\
$2010-1-1(55197.727)$ & HIP 55731 & Clay/MIKE & 36 & ISM Standard \\
$2010-1-1(55197.733)$ & HIP 55864 & Clay/MIKE & 40 & ISM Standard \\
$2010-1-1(55197.738)$ & HIP 55901 & Clay/MIKE & 100 & ISM Standard \\
$2010-1-1(55197.743)$ & HIP 55968 & Clay/MIKE & 47 & ISM Standard \\
$2010-6-17(55365.467)$ & HIP 55731 & Clay/MIKE & 78 & ISM Standard \\
$2010-6-17(55365.472)$ & HIP 55901 & Clay/MIKE & 162 & ISM Standard \\
$2010-6-17(55365.479)$ & HIP 55864 & Clay/MIKE & 105 & ISM Standard \\
$2010-6-17(55365.486)$ & WD 1124-293 & Clay/MIKE & 73 & \\
$2010-6-17(55365.575)$ & HIP 55968 & Clay/MIKE & 56 & ISM Standard \\
$2010-7-07(55384.500)$ & WD 1124-293 & Clay/MIKE & 33 & \\
$2011-6-09(55385.493)$ & WD 1124-293 & Clay/MIKE & 70 & \\
\hline & & & & \\
\hline
\end{tabular}


Table 2. Properties of Photospheric and Circumstellar Ca II lines in WD 1124-293

\begin{tabular}{|c|c|c|c|c|c|}
\hline Line type & JD-5400000 & $\begin{array}{c}\text { Ca II K EQW } \\
(\mathrm{m \AA})\end{array}$ & $\begin{array}{c}\text { Ca II H EQW } \\
(\mathrm{m} \AA)\end{array}$ & $\begin{array}{l}\mathrm{Ca} \text { II } \mathrm{K} \mathrm{v}_{r} \\
\left(\mathrm{~km} \mathrm{~s}^{-1}\right.\end{array}$ & $\begin{array}{c}\text { Ca II H v } \\
\left(\mathrm{km} \mathrm{s}^{-1}\right)\end{array}$ \\
\hline \multirow[t]{16}{*}{ Photospheric } & 51159.629 & $104 \pm 3$ & $39 \pm 4$ & 30.3 & 31.3 \\
\hline & 51288.328 & $98 \pm 5$ & $57 \pm 6$ & 28.7 & 27.5 \\
\hline & 51657.663 & $111 \pm 6$ & $58 \pm 12$ & 29.1 & 28.0 \\
\hline & 51681.588 & $109 \pm 17$ & $\ldots$ & 29.8 & $\ldots$ \\
\hline & 54189.682 & $106 \pm 4$ & $34 \pm 4$ & 29.7 & 34.5 \\
\hline & 54546.605 & $116 \pm 2$ & $52 \pm 3$ & 29.7 & 29.8 \\
\hline & 54547.658 & $113 \pm 2$ & $54 \pm 3$ & 29.0 & 29.4 \\
\hline & 54599.536 & $113 \pm 2$ & $53 \pm 2$ & 28.3 & 28.3 \\
\hline & 54600.551 & $114 \pm 3$ & $49 \pm 3$ & 28.6 & 28.8 \\
\hline & 54601.477 & $113 \pm 2$ & $55 \pm 2$ & 29.0 & 30.1 \\
\hline & 54602.502 & $118 \pm 2$ & $55 \pm 2$ & 29.2 & 29.4 \\
\hline & 54937.686 & $113 \pm 3$ & $53 \pm 3$ & 28.8 & 27.7 \\
\hline & 54969.548 & $127 \pm 3$ & $43 \pm 3$ & 29.1 & 27.8 \\
\hline & 55364.486 & $115 \pm 2$ & $52 \pm 1$ & 28.7 & 29.5 \\
\hline & 55385.493 & $95 \pm 3$ & $59 \pm 4$ & 28.4 & 29.7 \\
\hline & 55721.598 & $111 \pm 2$ & $54 \pm 2$ & 29.7 & 29.4 \\
\hline \multirow[t]{4}{*}{ Circumstellar } & March 2008 & $11 \pm 2$ & $\ldots$ & $-0.5 \pm 0.9$ & $\ldots$ \\
\hline & May 2008 & $12 \pm 1$ & $\ldots$ & $-2 \pm 1$ & $\ldots$ \\
\hline & June 2010+June 2011 & $10 \pm 1$ & $\ldots$ & $-1 \pm 1$ & $\ldots$ \\
\hline & Total & $11 \pm 1$ & $2 \pm 0.5$ & $-0.9 \pm 0.5$ & $-0.4 \pm 0.5$ \\
\hline
\end{tabular}




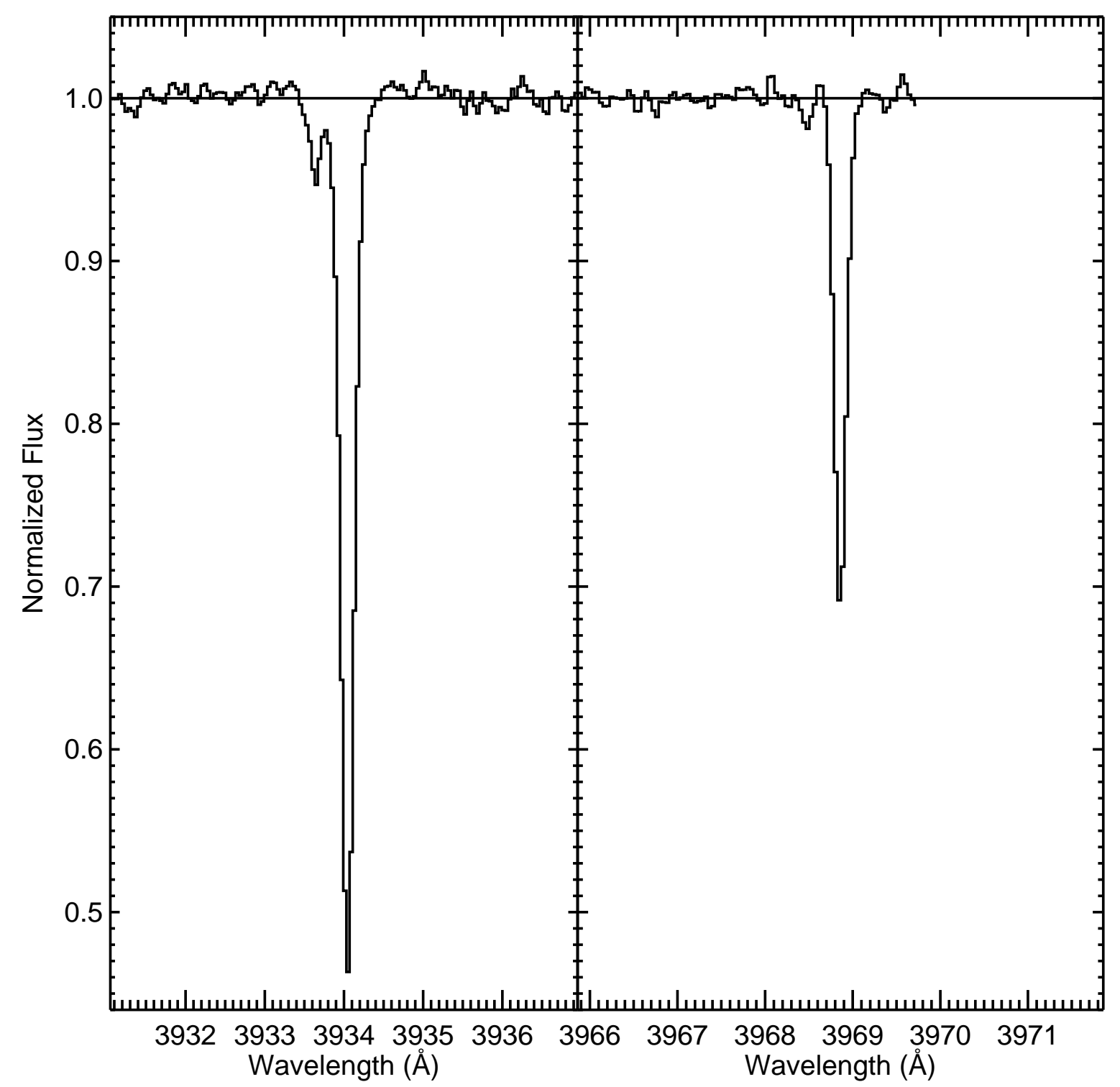

Fig. 1.- Detection of weak circumstellar Ca II features in the spectrum of WD 1124-293. The spectrum is from a $\mathrm{S} / \mathrm{N}$ weighted combination of observations spanning four years, and has a $\mathrm{S} / \mathrm{N}$ of 180 . The photospheric line is located at $\approx 3934 \AA$ and the circumstellar feature is blue shifted from the photospheric line. 


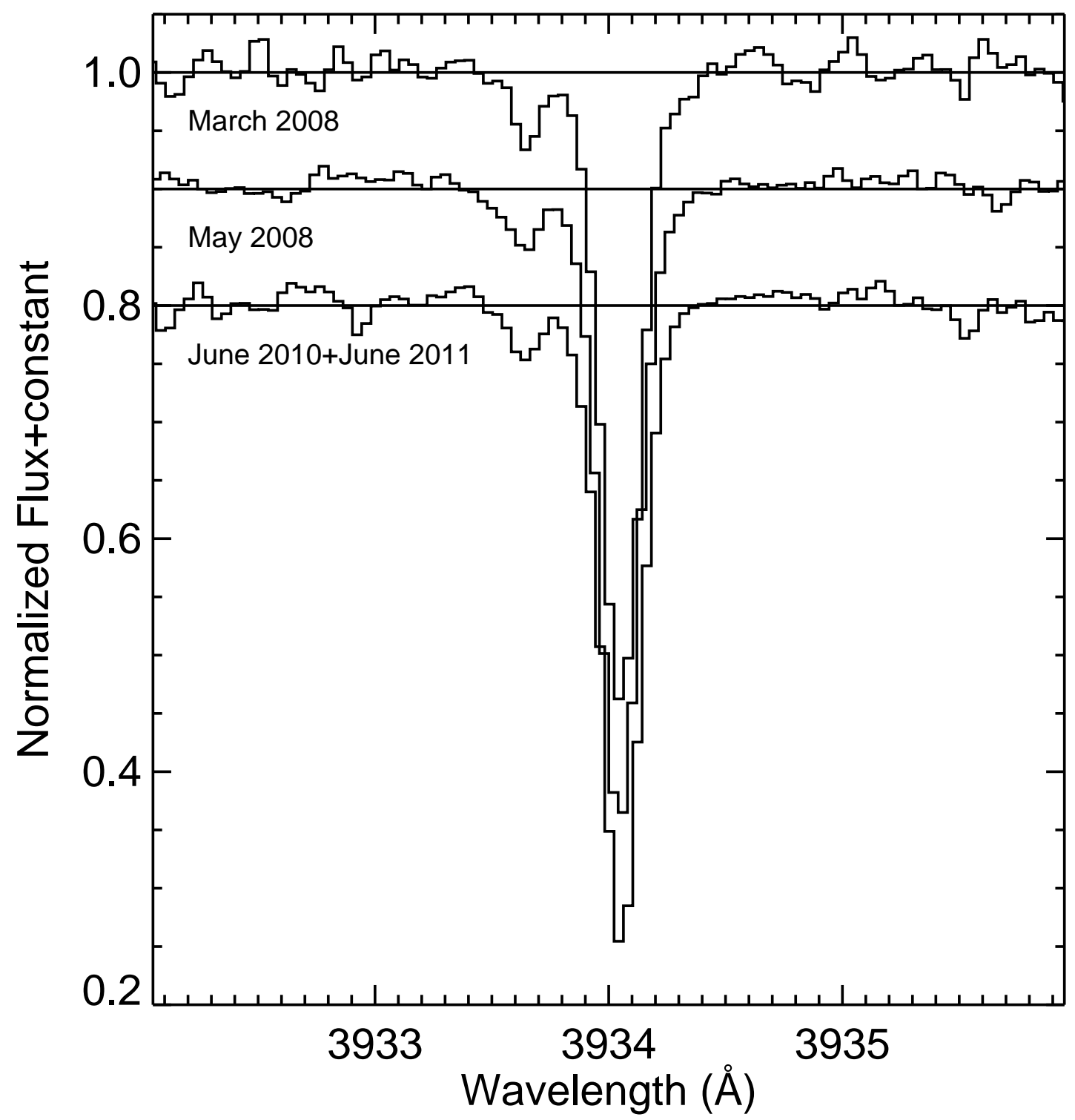

Fig. 2.- Three separate epochs which show a clear detection of the blueshifted weak Ca II feature. The line is recovered relative to the continuum with a significance of 4.7-, 4.9-, and 4- $\sigma$ for March 2008, May 2008, and June 2010+June 2011 respectively. 


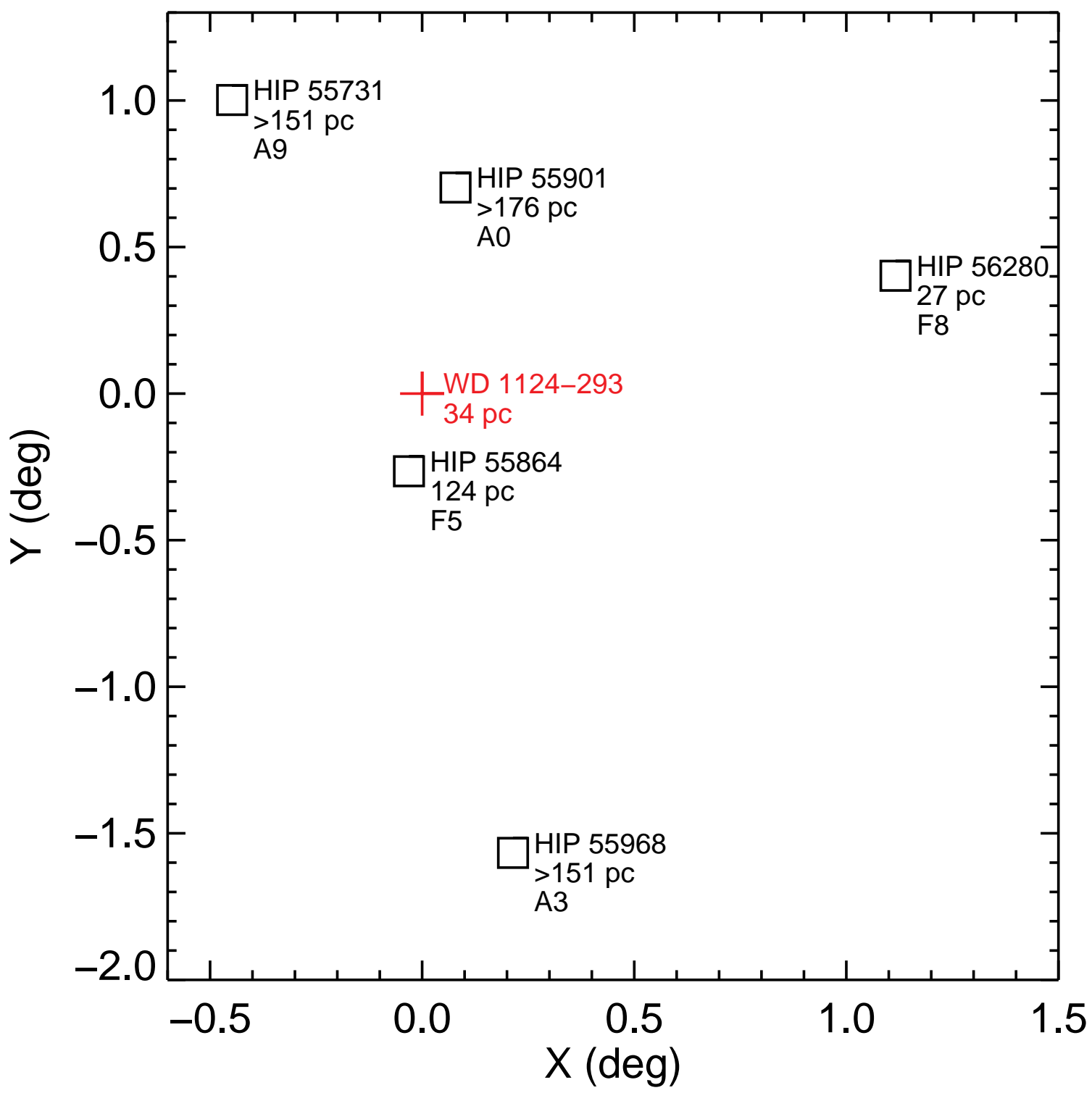

Fig. 3.- Location of ISM standards on the sky relative to WD 1124-293. HIP 55864 is the closest star to WD 1124-293 and is at a projected separation of $0.16 \mathrm{pc}$ at the distance to the WD. 

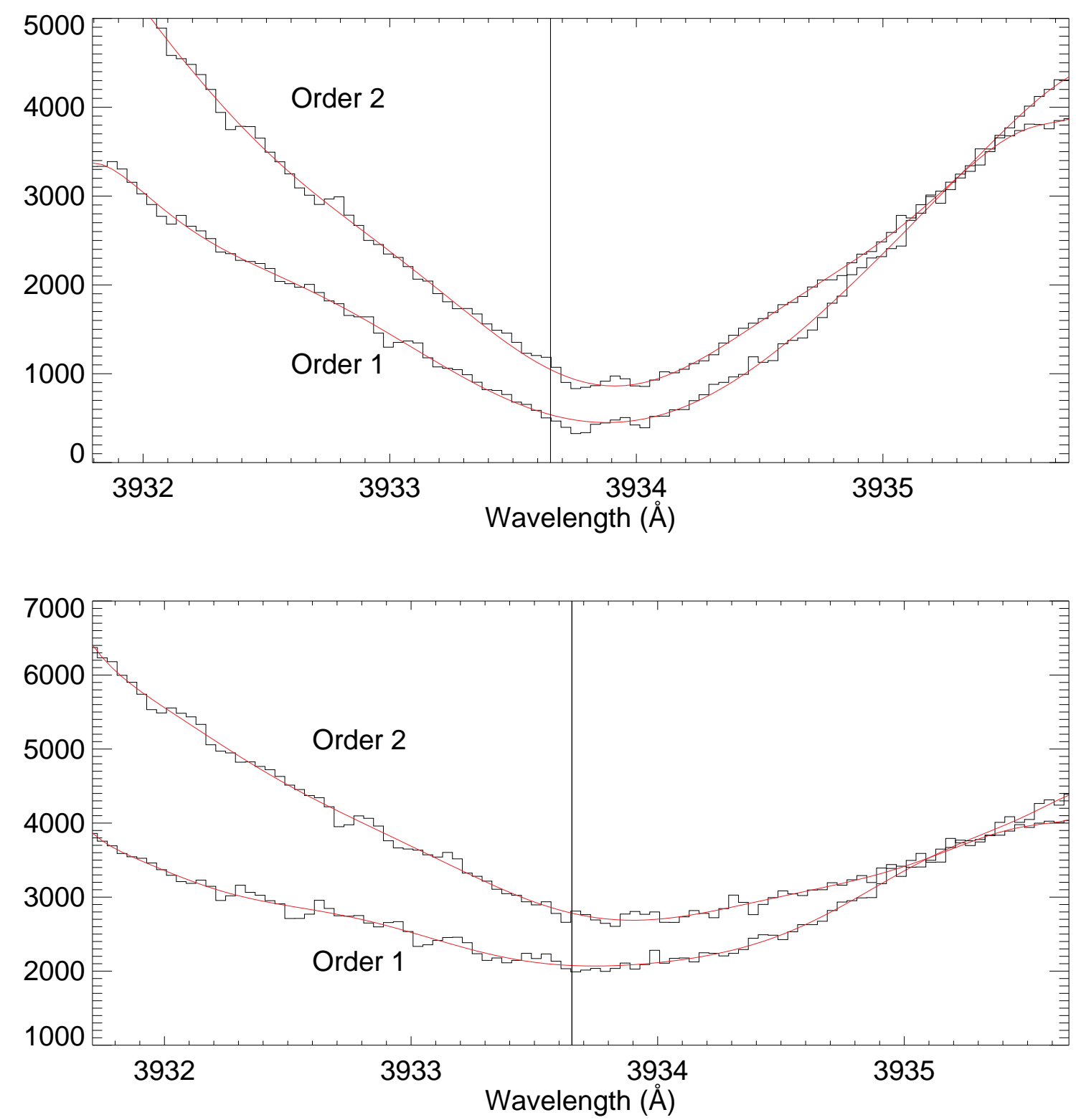

Fig. 4.- Comparison of polynomial fitting of the broad and deep photospheric Ca II lines of two ISM standards, HIP 55731 and HIP 55864. The histograms are the observed spectra in each order of the MIKE echelle spectrum while the solid lines are the fits. The solid vertical line marks the location of WD 1124-293's circumstellar feature. 


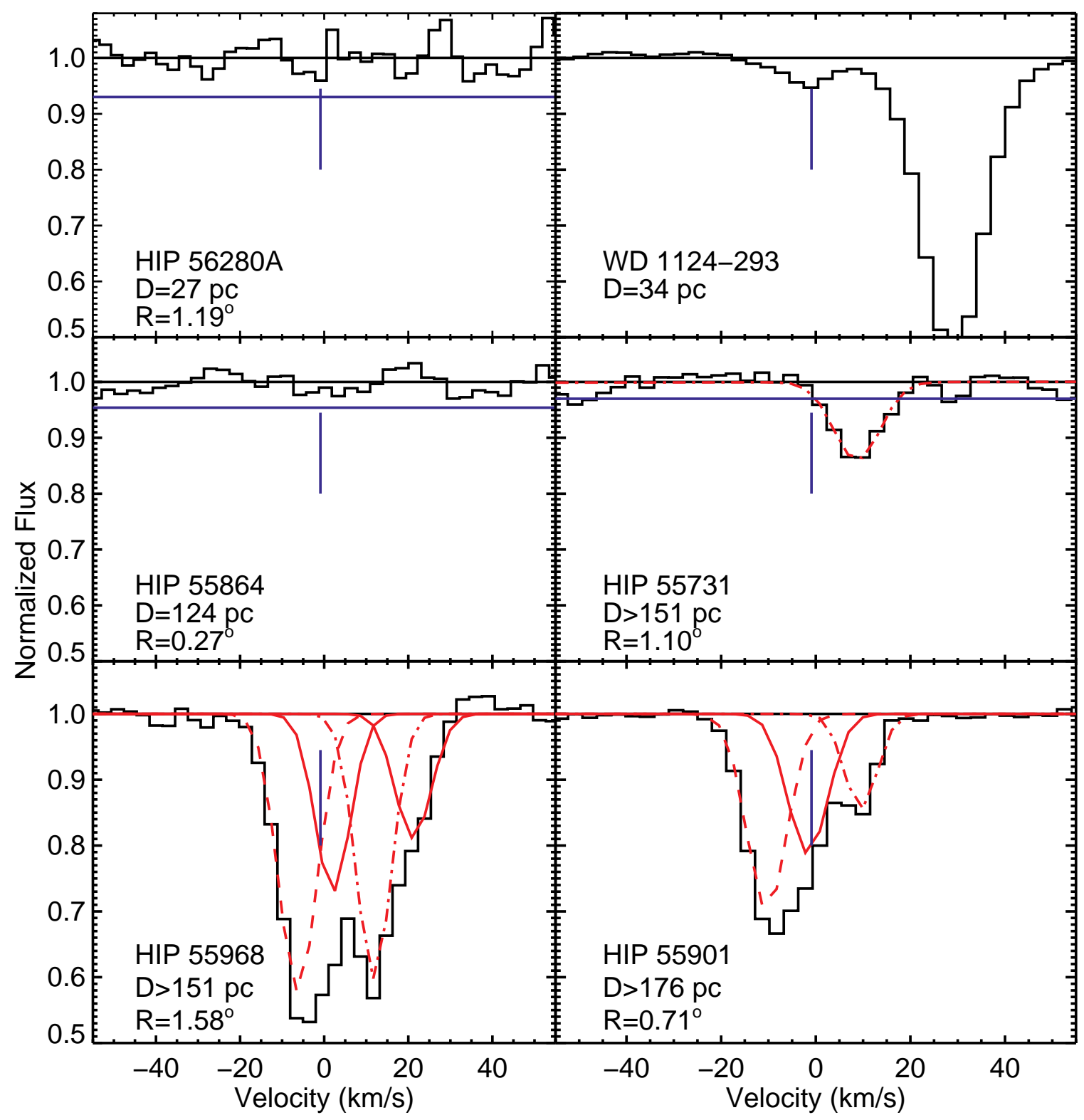

Fig. 5. - Comparison of the location of the weak Ca II K feature in WD 1124-293 with our ISM standards, arranged by probable distance from Earth. The dark vertical line shows the location of the weak circumstellar feature of WD 1124-293, while the horizontal blue line shows the 3- $\sigma$ detection threshold for standards that showed no absorption at the wavelength of WD 1124-293's component. More distant standards show ISM absorption, while standards closer than $124 \mathrm{pc}$ show no appreciable ISM absorption, ruling out LISM as a source for WD 1124-293's blueshifted feature. 


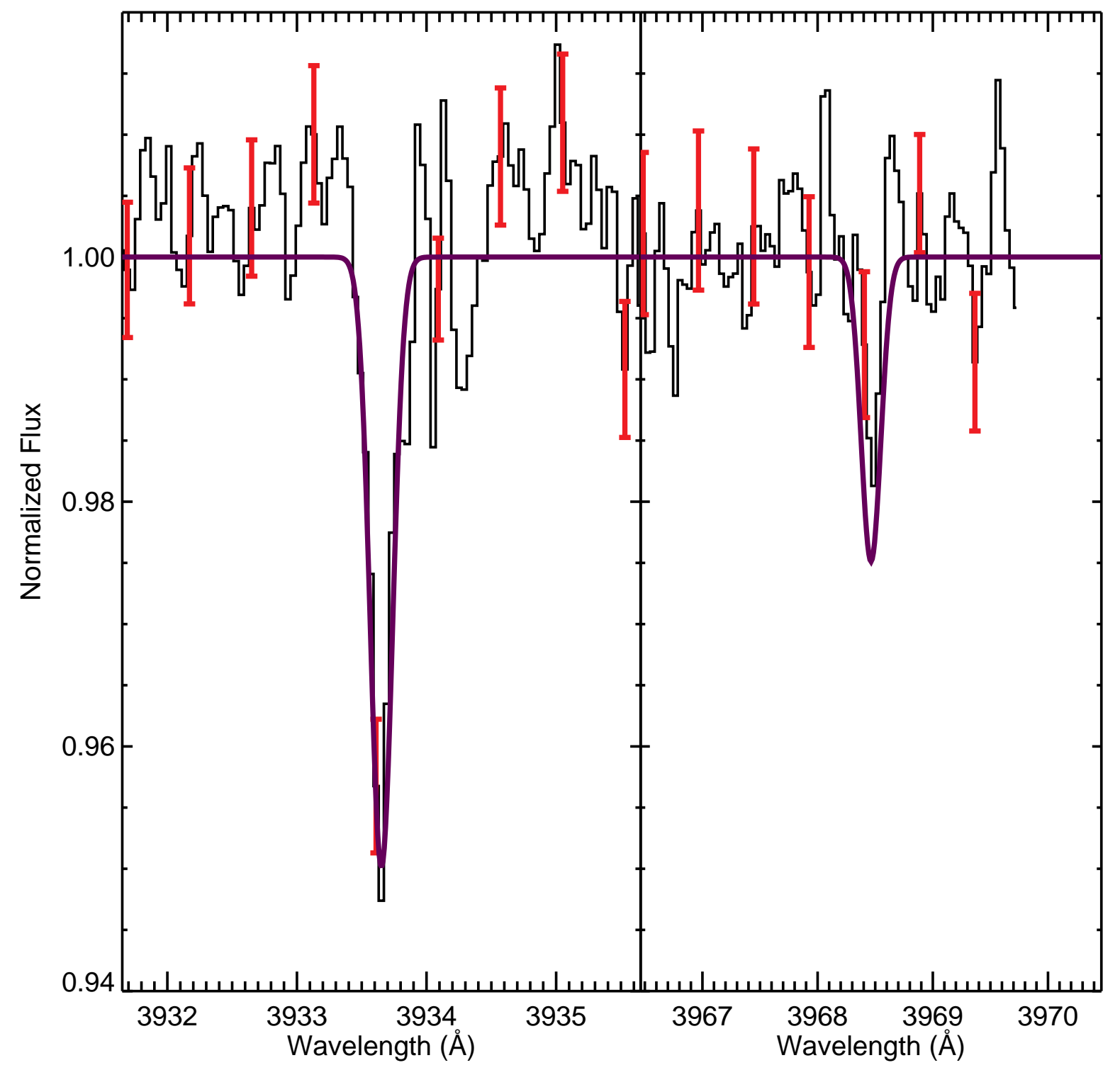

Fig. 6. - Comparison of the observed circumstellar Ca line (black histogram with red error bars) with the photospheric line divided out to a model Ca II cloud (solid dark line) with a column density of $\log N=11.07_{-0.2}^{+0.1}$. 

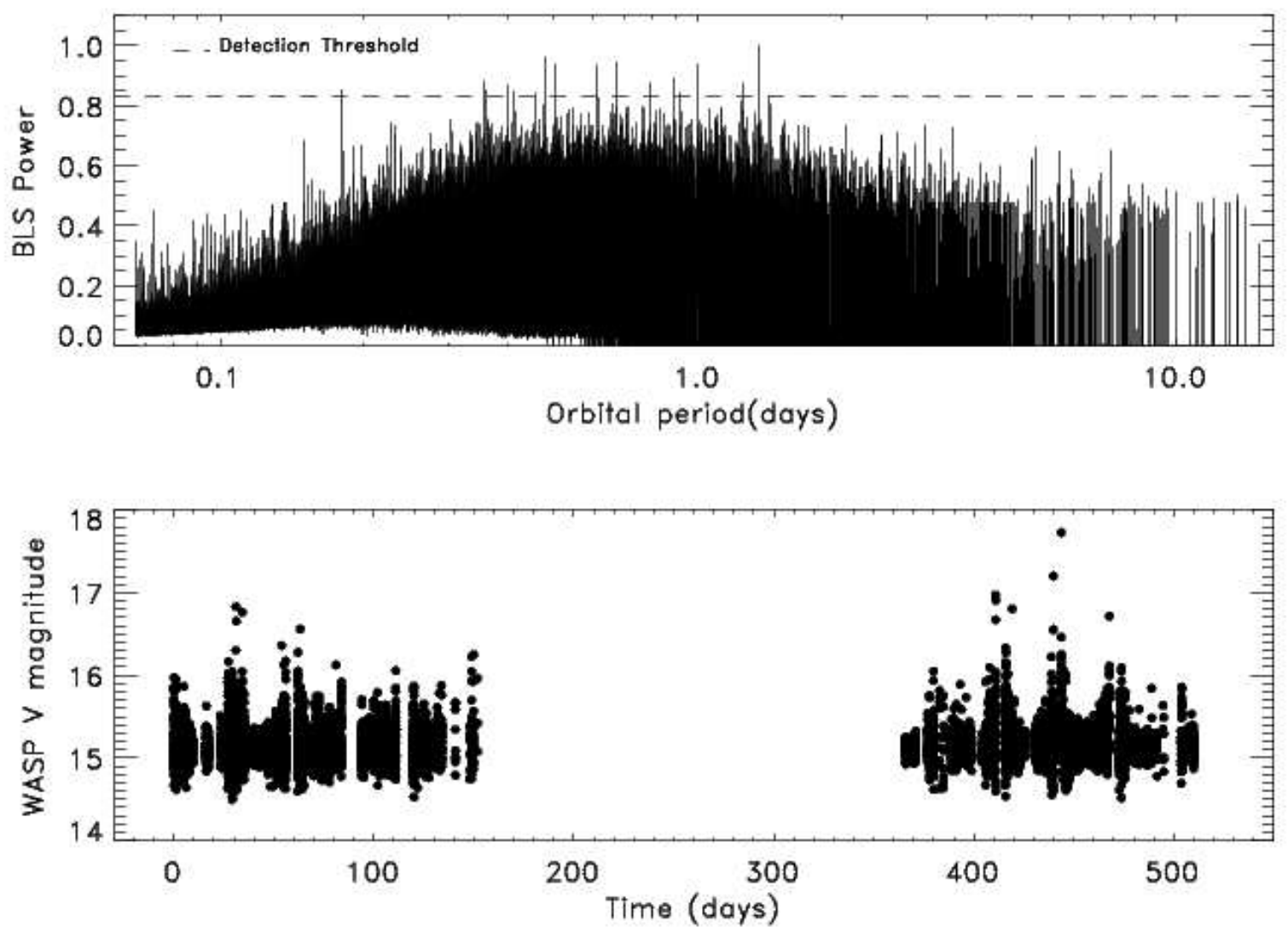

Fig. 7.- Top panel: Box-Least Square power spectrum for the white dwarf WD1124-293. Lower panel: WASP light-curve for WD1124-293 covering the period January 2007 - May 2008, with a total of 10618 photometric measurments. We used WASP V-magnitude, where $\mathrm{V}_{\text {mag }}=-2.5 * \log \left(\right.$ flux $\left./ 10^{6}\right)$, expressed in $\mu$ Vega. 
Table 3. Properties of ISM clouds for ISM standards

\begin{tabular}{|c|c|c|c|c|c|c|c|c|}
\hline \multirow[t]{2}{*}{ Name } & \multicolumn{2}{|c|}{ Cloud I } & \multicolumn{2}{|c|}{ Cloud 2} & \multicolumn{2}{|c|}{ Cloud 3} & \multicolumn{2}{|c|}{ Cloud 4} \\
\hline & $\begin{array}{c}\mathrm{v} \\
\left(\mathrm{km} \mathrm{s}^{-1}\right)\end{array}$ & $\begin{array}{c}\mathrm{EW} \\
(\mathrm{m} \AA)\end{array}$ & $\begin{array}{c}\mathrm{v} \\
\left(\mathrm{km} \mathrm{s}^{-1}\right)\end{array}$ & $\begin{array}{c}\mathrm{EW} \\
(\mathrm{m} \AA)\end{array}$ & $\begin{array}{c}\mathrm{v} \\
\left(\mathrm{km} \mathrm{s}^{-1}\right)\end{array}$ & $\begin{array}{c}\mathrm{EW} \\
(\mathrm{m} \AA)\end{array}$ & $\begin{array}{c}\mathrm{v} \\
\left(\mathrm{km} \mathrm{s}^{-1}\right)\end{array}$ & $\begin{array}{c}\mathrm{EW} \\
(\mathrm{m} \AA)\end{array}$ \\
\hline HIP 56280 & $\ldots$ & $<10$ & $\ldots$ & $<10$ & $\ldots$ & $<10$ & $\ldots$ & $<10$ \\
\hline HIP 55864 & $\ldots$ & $<7$ & $\ldots$ & $<7$ & $\ldots$ & $<7$ & $\ldots$ & $<7$ \\
\hline HIP 55731 & $\ldots$ & $<6$ & $\ldots$ & $<6$ & 8.7 & $22 \pm 2$ & $\ldots$ & $<6$ \\
\hline HIP 55901 & -10.3 & $47 \pm 1$ & -1.7 & $34 \pm 1$ & 9.5 & $17 \pm 1$ & $\ldots$ & $<2$ \\
\hline HIP 55968 & -6.3 & $72 \pm 2$ & 2.0 & $43 \pm 2$ & 11.8 & $56 \pm 2$ & 21.2 & $19 \pm 2$ \\
\hline
\end{tabular}

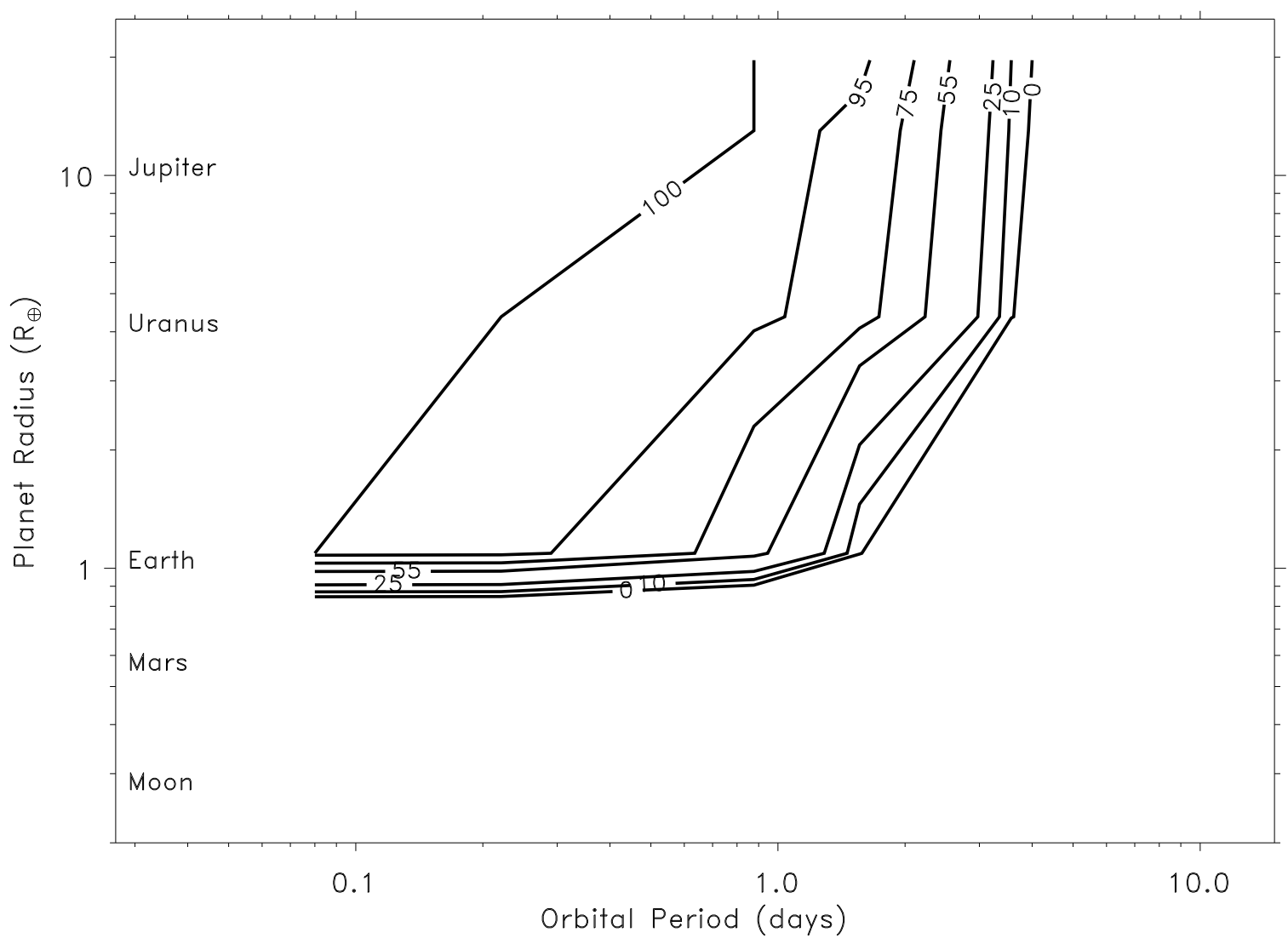

Fig. 8.- Detection limits for planetary companions of sizes ranging from Jupiter-size to Moon-size in orbit around the WD1124-293. The numbers on the contours relate to the detection efficiency in percentage. 
Table 4. Recovery rate of simulated transits of WD1124-293

\begin{tabular}{ccccc}
\hline \hline Size & $\mathrm{R}_{\mathrm{p}}$ & $\delta_{\text {tr }}$ & $\mathrm{P}$ & $\mathrm{f}_{\mathrm{det}}$ \\
& $\mathrm{R}_{\oplus}$ & $(\%)$ & $($ days $)$ & $(\%)$ \\
& & & & \\
\hline Jupiter & \multirow{2}{*}{10.0} & 100 & 0.08 & 100 \\
& & & 0.22 & 100 \\
& & & 0.87 & 100 \\
& & & 1.56 & 91 \\
& & & 3.57 & 8 \\
& & & & 0 \\
Uranus & 4.0 & 100 & 0.08 & 100 \\
& & & 0.22 & 100 \\
& & & 0.87 & 99 \\
& & & 1.56 & 82 \\
& & & 3.57 & 1 \\
& & & & \\
Earth & 1.0 & 49 & 0.08 & - \\
& & & 0.22 & 99 \\
& & & 0.87 & 61 \\
& & & 1.56 & 1 \\
& & & 3.57 & 0 \\
\hline
\end{tabular}




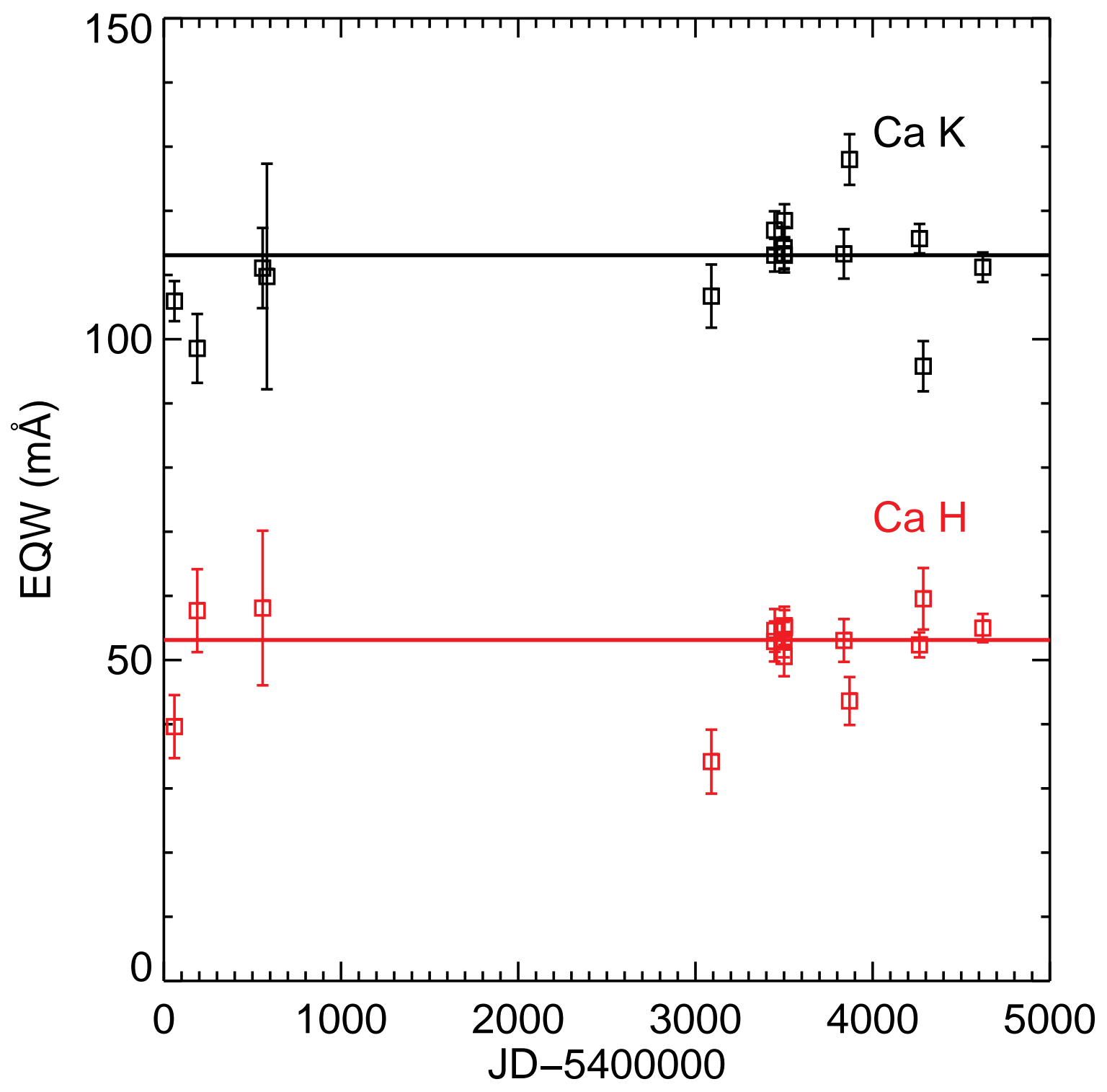

Fig. 9. - The EW of Ca II in WD 1124-293's photosphere as a function of time. The EWs are compared to the expectation of a constant value, and do not significantly vary. Neglecting spurious points, the largest epoch-to-epoch variations are less than $7 \%$ and $5 \%$ from the median $\mathrm{EW}$ value for the $\mathrm{Ca} \mathrm{H}$ (lower points) and $\mathrm{K}$ (upper points) lines respectively. 